\title{
Structural and functional studies of key enzyme (LpxC (UDP-3-O-acyl-N-acetylglucosamine deacetylase) from salmonella typhi involved in Gram-negative bacterial lipid A biosynthesis
}

\author{
Sudhir Kumar Pal, Sanjit Kumar \\ Centre for Bio Separation Technology, VIT University, Katpadi, Vellore, Tamil Nadu-632014 \\ sudhirkumarpal001@gmail.com
}

Over the last decade, in the case of gram-negative bacteria the frequency of antibacterial resistance especially in ill and hospitalized patients (including multidrug resistance (MDR) and its association with severe infectious diseases) has increased at alarming rates. Salmonella typhi (gram negative bacteria) is an ubiquitous pathogen responsible for a number of diseases such as pneumonia, meningitis, etc. The increasing number of infections caused by MDR Salmonella typhi must be re-explored for novel treatment strategies. LpxC, a metal dependent amidase (highly active in the presence of $\mathrm{Zn} 2+$ ions $(\mathrm{Kd} \sim 60 \mathrm{pM})$ ) is one such vital and ratelimiting enzyme committing the step of Lipid A (a strong human immuno-modulator bacterial endotoxin) biosynthesis. Many structures of LpxC enzyme in complex with different inhibitors are solved by various structural biology techniques but most of these inhibitors have been reported to have poor anti-microbial activity. Compounds with indole-2-carboxamide scaffold in their skeleton display various biological activities including anti-bacterial activity. We have done indole-2-carboxamide scaffold-based virtual screening and observed inhibitors like $0435(-9.0 \mathrm{kcal} / \mathrm{mol}), 0436(-9 \mathrm{kcal} / \mathrm{mol}), 1812(-8.6 \mathrm{kcal} / \mathrm{mol}), 2584(-8.5 \mathrm{kcal} / \mathrm{mol})$, and 2545 $(-8.4 \mathrm{kcal} / \mathrm{mol})$ bound to the functional active site of $\mathrm{LpxC}$.

Keywords: Multidrug Resistance, LpxC, Salmonella typhi, Lipid A, inhibition 